\title{
Upregulation of the RNF8 gene can predict the presence of sperm in azoospermic individuals
}

\author{
Majid Nazari', Emad Babakhanzadeh',2, S. Mohsen Aghaei Zarch'1, Mehrdad Talebi', Nima Narimani , Mandana Dargahi', \\ Marjan Sabbaghian ${ }^{5}$, Nasrin Ghasemi ${ }^{6}$ \\ 'Department of Medical Genetics, ${ }^{2}$ Yazd Medical Genetics Research Center, and ${ }^{3}$ Department of Urology, Research and Clinical Center for Infertility, \\ Shahid Sadoughi University of Medical Sciences, Yazd; ' ${ }^{4}$ Department of Pathology, Azad University of Medical Science, Yazd; ${ }^{5}$ Department of Andrology, \\ Reproductive Biomedicine Research Center, Royan Institute for Reproductive Biomedicine, ACECR, Tehran; ${ }^{6}$ Abortion Research Center, Yazd \\ Reproductive Sciences Institute, Shahid Sadoughi University of Medical Sciences, Yazd, Iran
}

Objective: In this study, specimens from testicular biopsies of men with nonobstructive azoospermia (NOA) were used to investigate whether RNF8 gene could serve as a biomarker to predict the presence of sperm in these patients.

Methods: Testicular biopsy specimens from 47 patients were classified according to the presence of sperm (positive vs. negative groups) and investigated for the expression of RNF8. The level of RNF8 gene expression in the testes was compared between these groups using reversetranscription polymerase chain reaction.

Results: The expression level of RNF8 was significantly higher in testicular samples from the positive group than in those from the negative group. Moreover, the area under the curve of RNF8 expression for the entire study population was 0.84 , showing the discriminatory power of RNF8 expression in differentiating between the positive and negative groups of men with NOA. A receiver operating characteristic curve analysis showed that RNF8 expression had a sensitivity of $81 \%$ and a specificity of $84 \%$, with a cutoff level of 1.76 .

Conclusion: This study points out a significant association between the expression of RNF8 and the presence of sperm in NOA patients, which suggests that quantified RNF8 expression in testicular biopsy samples may be a valuable biomarker for predicting the presence of spermatozoa in biopsy samples.

Keywords: Azoospermia; Biomarker; RNF8; Spermatogenesis

\section{Introduction}

Infertility, which is defined as a disability to conceive a child after regular unprotected intercourse over the course of 1 year, affects

Received: June 20, 2019· Revised: August 30, 2019· Accepted: September 5, 2019 Corresponding author: Nasrin Ghasemi

Abortion Research Center, Yazd Reproductive Sciences Institute, Shahid

Sadoughi University of Medical Sciences, Yazd 8916877391, Iran

Tel: +98-03538247085 Fax:+98-03538248087

E-mail:nghasemi479@gmail.com

*This experiment was supported by Shahid Sadoughi University of Medical Sciences.

This is an Open Access article distributed under the terms of the Creative Commons Attribution Non-Commercial License (https://creativecommons.org/licenses/by-nc/4.0/) which permits unrestricted non-commercial use, distribution, and reproduction in any medium, provided the original work is properly cited.
$13 \%-18 \%$ of couples globally [1]. Male factors account for infertility in roughly half of all cases [2], and azoospermia is the most common male factor leading to infertility [3]. Azoospermia can be concisely described as the absence of sperm in the ejaculate, and it is classified into obstructive azoospermia $(\mathrm{OA})$, which derives from a blockage in the genital tract, and nonobstructive azoospermia (NOA), which results from a failure of spermatogenesis [4]. In some NOA samples, germ cells are absent and the men are diagnosed as having Sertoli cell-only syndrome [5]. Men whose germ cells have no detectable mature spermatids are diagnosed with maturation arrest [6]. In other samples, spermatogenesis can sporadically progress to mature spermatids in some tubules, a process known as hypospermatogenesis [7].

If sperm can be retrieved by microdissection testicular sperm extraction (mTESE), men with NOA may be able to fertilize their partners by 
intracytoplasmic sperm injection (ICSI) [8]. The rate of sperm retrieval in testicular operations is about 50\% [9], and to date, the only generally accepted reliable predictor of successful mTESE is testicular histology [10]. Failure to retrieve sperm may have devastating consequence for couples who may not want to undergo sperm or embryo donation. Subsequent surgery after the first mTESE procedure with the hope of finding possible spermatogenic loci may be the best option for many of these couples. Since some researchers believe that spermatozoa retrieved from NOA patients are not appropriate for freezing [11], and a second or repeated mTESE procedure may therefore be considered in these patients for future ICSI, it seems necessary to find additional criteria based on a noninvasive approach in order to predict the success of sperm retrieval in patients with NOA.

RNF8 is an E3 ubiquitin ligase located on chromosome 6p21.3 that spans 8 exons. It plays important roles in many biological processes, including DNA repair $[12,13]$ and chromatin remodeling [14]. Ubiquitination of histones in the testes by RNF8 leads to nucleosomal replacement by protamines, which is essential for the condensation of chromatin and spermatogenesis [15]. A lack of RNF8 in mice leads to infertility due to defective spermiogenesis [16], and RNF8-knockout mice are unable to generate mature sperm [17]. Additionally, through RNF8dependent mechanisms, sex-linked genes (including the Ssty gene family) escape post-meiotic silencing and are activated in round spermatids and throughout the course of spermiogenesis $[18,19]$. Downregulation of the Ssty gene family was found to be correlated with a reduced amount of sperm in the testes of mice [20]. These studies indicate that RNF8 plays an essential role in modulating mechanisms related to spermatogenesis and that its absence could result in infertility.

\section{Methods}

\section{Patients}

Forty-seven men with OA and NOA who underwent mTESE to obtain spermatozoa for ICSI were enrolled in this study. The patients were chosen based on the availability of well-preserved testicular biopsy samples for research. The Institutional Ethics Committee of Shahid Sadoughi Medical University approved this study (IRB No. 1396.219) and written informed consent was obtained from all patients before the collection of tissue samples. The preoperative examination included history, measurement of testis size, semen analysis, and quantification of serum follicle-stimulating hormone (FSH), luteinizing hormone (LH), and testosterone levels. Moreover, no patients were on hormonal therapy and all had primary infertility. None of the participants had a history of TESE or cryptorchidism. Patients with cystic fibrosis or chromosomal abnormalities such as Klinefelter syndrome and $\mathrm{Y}$ chromosome microdeletion were excluded from the study.

\section{Tissue acquisition and histological analysis}

Spermatozoa were obtained by testicular biopsy. The pieces of testicular tissue weighing approximately $20 \mathrm{mg}$ that were used for RNA isolation were placed into a cryovial and instantly snap-frozen in liquid nitrogen. Depending on whether sperm were recovered, the azoospermic patients were divided into OA and NOA groups. Sperm retrieval was considered to be positive if, in the laboratory, at least one spermatozoon was found that was morphologically suitable for ICSI. Additionally, another small piece of testicular tissue was paraffin-embedded and fixed using Bouin solution for histopathological evaluation. After staining with hematoxylin and eosin, biopsy samples were evaluated as described elsewhere [21].

\section{RNA extraction and cDNA synthesis}

Total RNA was extracted from the tissue samples using RNeasy Plus Universal (Qiagen, Hilden, Germany) based on the manufacturer's protocol and kept at $-80^{\circ} \mathrm{C}$. The concentration and purity of the isolated RNA were determined by the A260/A280 ratio using a Nanodrop 2000 spectrophotometer (Thermo Scientific, Wilmington, DE, USA) and confirmed by agarose gel electrophoresis. Template CDNA was synthesized from $1 \mu \mathrm{g}$ of whole extracted RNA with a Revert Aid First Strand cDNA Synthesis Kit (Thermo Scientific, Vilnius, Lithuania) using oligo-dT and random hexamer primers simultaneously for each reaction in an Eppendorf Mastercycler Gradient device (Hamburg, Germany).

\section{Quantitative reverse-transcription polymerase chain reaction and primer design}

For reverse-transcription polymerase chain reaction (RT-PCR), the initial denaturation step took 10 minutes, followed by 35 cycles of denaturation at $94^{\circ} \mathrm{C}$ for 30 seconds, annealing at $59^{\circ} \mathrm{C}$ for 30 seconds, and extension at $72^{\circ} \mathrm{C}$ for 30 seconds. The melting curve was derived by increasing the temperature from $72^{\circ} \mathrm{C}$ to $95^{\circ} \mathrm{C}$ to guarantee that no genomic DNA or primer dimers were amplified in the reactions. Quantitative PCR (qPCR) was run in triplicates on 48-well plates (Applied Biosystems, Step One Plus, Foster City, CA, USA) and the average CT value was used for further analysis. All RT-PCR runs contained nontemplate (CDNA) controls in order to avoid potential contamination. Relative gene expression analysis was performed using the comparative CT method $\left(2^{\Delta \Delta C T}\right)$. The $2^{\Delta \Delta C T}$ parameter represents the expression of a gene of interest (here, RNF8) as a fold multiple with respect to the housekeeping ACTB gene. To design the primers, the following criteria were applied consecutively: mutual exons were retrieved between all transcripts from Ensembl, primers were chosen for exon-exon junction sites so that 6-10 nucleotides at the $3^{\prime}$ end of the primer sequence would hybridize to 1 exon and 10-16 nucleotides at the $5^{\prime}$ end would bind to the adjacent exon, the dynamic and structural fea- 
tures of primers were evaluated using OligoAnalyzer, the primers' specificity was verified by NCBI BLAST, and Ensembl was used to ensure that no polymorphism existed (with a minor allele frequency lower than 0.01 ) at the last base of the $3^{\prime}$ end of the primer sequence. The features of the primers are presented in Table 1. Lowercase base pairs are located at the $3^{\prime}$ end of the primer sequence.

Table 1. Real-time RT-PCR primers used in this study

\begin{tabular}{llcc}
\hline Gene & \multicolumn{1}{c}{ Primer sequencing $\left(5^{\prime} \rightarrow 3^{\prime}\right)$} & $\begin{array}{c}\text { Product size } \\
(\mathrm{bp})\end{array}$ & $\begin{array}{c}\text { Annealing } \\
\text { temperature }\left({ }^{\circ} \mathrm{C}\right)\end{array}$ \\
\hline RNF8 & F: GATGGGTGCGAGgtgactg & 153 & 59 \\
& $\begin{array}{l}\text { R: CCACCATTTAGACTcttgttgtcc } \\
\text { ACTB }\end{array}$ & & \\
& F: CCTTCCTGGgcatggag & 204 & 57 \\
& R: CGGAGCAATGATcttgatctt & & \\
\hline
\end{tabular}

RT-PCR, reverse-transcription polymerase chain reaction; $F$, forward; $R$, reverse.

\section{Statistical analysis}

Serum LH and FSH levels, testosterone levels, average testicular volume, age, and mean RNF8 expression were examined with respect to the mTESE results using the independent $t$-test, with $p$-values $<0.05$ considered to indicate statistical significance. Comparisons among the $\mathrm{OA}, \mathrm{NOA}-$, and $\mathrm{NOA}+$ groups were made by utilizing one-way analysis of variance followed by the Dunnett multiple comparison post-test. A receiver operating characteristic $(\mathrm{ROC})$ curve was constructed to predict the presence of retrievable sperm based on RNF8 expression. All analyses were performed in IBM SPSS ver. 23.0 (IBM Corp., Armonk, NY, USA).

\section{Results}

As shown in Table 2, sperm retrieval was successful in 19 of the 40

Table 2. Clinical characteristics of the patients

\begin{tabular}{|c|c|c|c|c|}
\hline Variable & $\mathrm{OA}$ & $\mathrm{NOA}+$ & NOA- & $p$-value \\
\hline Number & 7 & 19 & 21 & - \\
\hline Age (yr) & $29.0 \pm 4.6$ & $30.2 \pm 1.7$ & $33.1 \pm 3.5$ & - \\
\hline Testicular volume (mL) & $14.1 \pm 1.2$ & $10.9 \pm 4.6$ & $9.7 \pm 3.1$ & $<0.001$ \\
\hline $\mathrm{LH}(\mathrm{mlU} / \mathrm{mL})$ & $5.1 \pm 0.4$ & $8.9 \pm 0.5$ & $13.4 \pm 1.2$ & $<0.001$ \\
\hline $\mathrm{FSH}(\mathrm{mlU} / \mathrm{mL})$ & $8.9 \pm 0.4$ & $9.2 \pm 2.4$ & $15.9 \pm 1.1$ & $<0.001$ \\
\hline Testosterone (ng/mL) & $3.6 \pm 0.1$ & $4.1 \pm 0.9$ & $5.0 \pm 1.3$ & NS \\
\hline Transcript expression & $1.33 \pm 0.17$ & $1.61 \pm 0.23$ & $1.9 \pm 0.42$ & $<0.001$ \\
\hline
\end{tabular}

Values are presented as mean \pm standard deviation.

OA, obstructive azoospermia; NOA, nonobstructive azoospermia; +, presence of sperm; -, absence of sperm; LH, luteinizing hormone; FSH, follicle-stimulating hormone; NS, not significant.
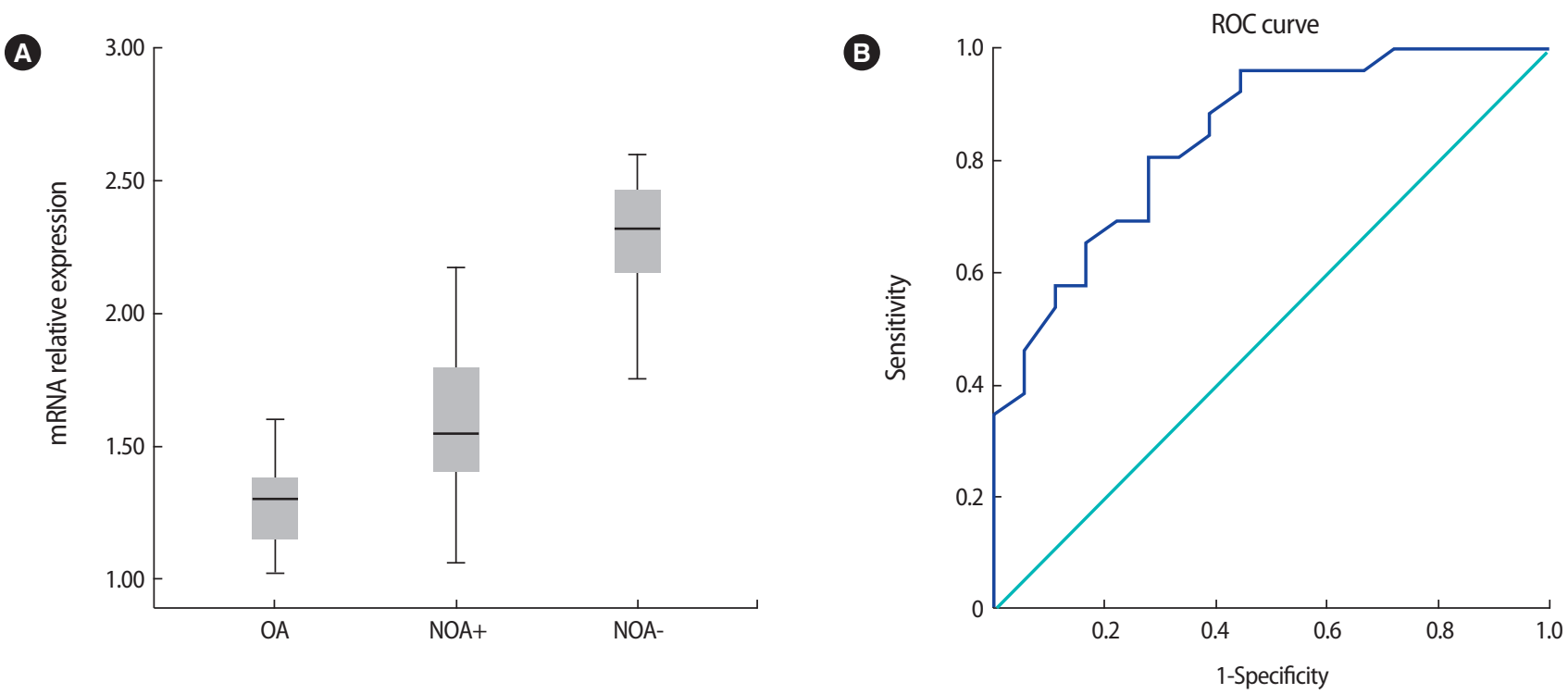

Figure 1. (A) Differential expression of the RNF8 gene in the obstructive azoospermia (OA), nonobstructive azoospermia (NOA)+ (presence of sperm), and NOA-(absence of sperm) groups. ACTB was used as a housekeeping gene. Each reaction was performed in triplicate. Expression of $R N F 8$ was upregulated in the NOA- group $(2.27 \pm 0.18)$ compared with the NOA+ $(1.6 \pm 0.23)$ and OA (1.3 \pm 0.17$)$ groups. The difference was significant $(p=0.001)$. (B) Receiver operating characteristic (ROC) curve of RNF8 expression in predicting sperm recovery outcomes. 

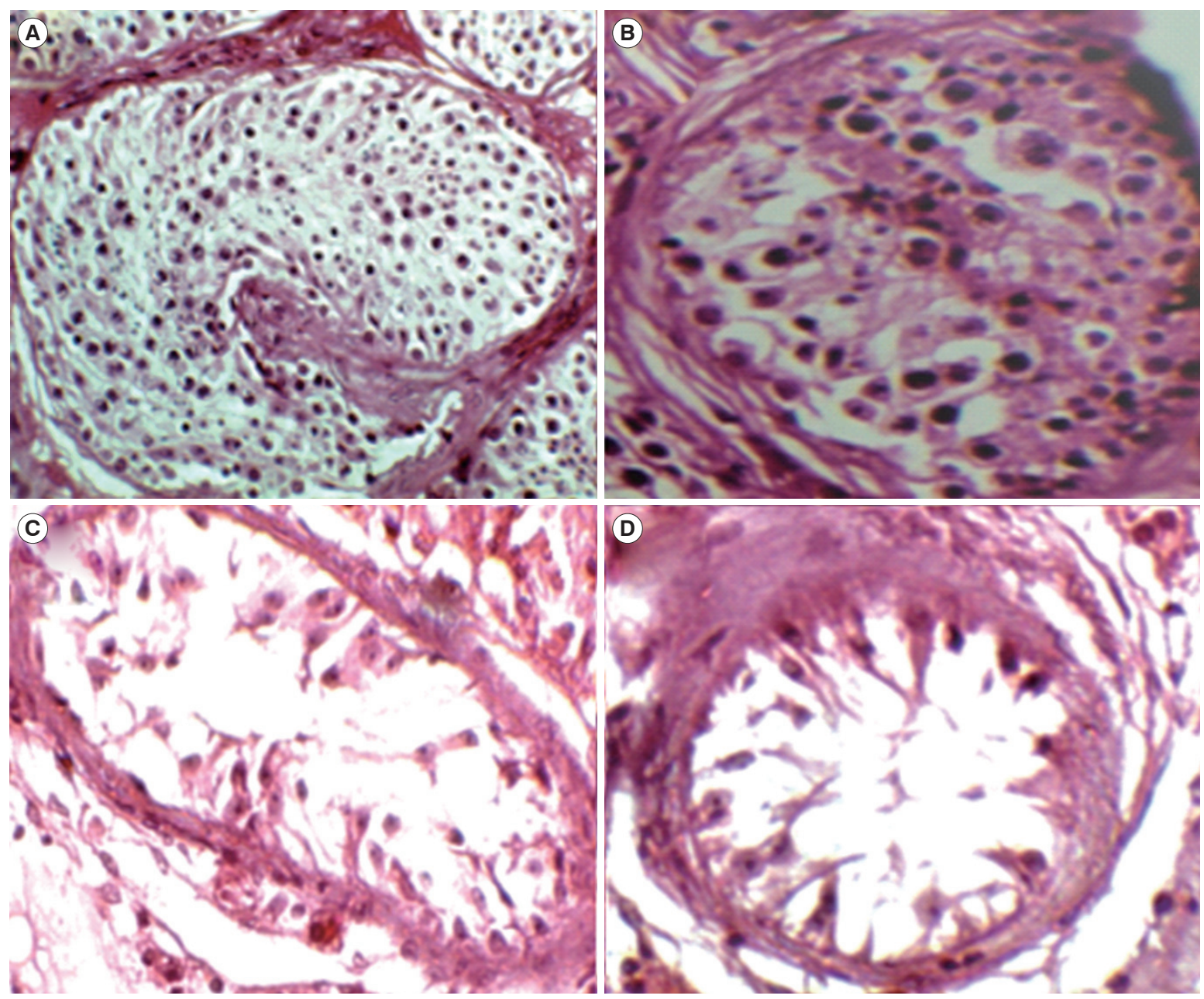

Figure 2. H\&E staining of histological samples from men with azoospermia. Specimens were categorized into the following four groups: (A) obstructive azoospermia, (B) hypospermatogenesis, (C) maturation arrest, and (D) Sertoli cell-only.

men with NOA. There were no significant differences in mean age and testosterone levels between the $\mathrm{OA}, \mathrm{NOA}+$, and NOA- groups. The NOA- patients showed the highest mean FSH and LH levels. In addition, testicular volume had a lower mean value in the NOAgroup than in the NOA+ and OA groups (Table 2). The level of RNF8 mRNA expression in testicular biopsy samples was assessed using $\mathrm{qPCR}$, and as illustrated in Figure $1 \mathrm{~A}$, it was significantly higher in the $\mathrm{NOA}-$ group than in the NOA+ and OA groups $(p<0.001)$. As shown in Figure 2, histopathological analyses revealed that 12, 15, and 13 individuals had Sertoli cell-only syndrome, maturation arrest, and hypospermatogenesis, respectively.

The diagnostic power of RNF8 expression was evaluated based on the area under the curve (AUC), and its specificity and sensitivity were determined based on the ROC curve. To discriminate azoospermic patients with and without successful sperm recovery, the optimal cutoff point was determined to be 1.76, which yielded an AUC of 0.84 (95\% confidence interval, $0.764-0.974 ; p<0.001)$ with sensitivi- ty and specificity values of 0.80 and 0.83 , respectively (Figure 1B).

\section{Discussion}

Due to the heterogeneous nature of the testis, researchers have struggled to find a definitive marker capable of predicting the presence of spermatozoa in the testis [16]. Many noninvasive assessments have been used to predict the likelihood of sperm retrieval in NOA patients, including histological analyses, testicular volume measurements, assessments of hormone levels, and semen analysis; however, these techniques have not shown sufficient predictive power to produce reliable results $[22,23]$. Only patients who have been diagnosed with complete loss of the AZFa or AZFb regions are definitively considered to have no spermatozoa in their testicular biopsy samples, meaning that it is recommended to avoid mTESE in those patients [18]. More recently, it has been suggested that alterations of molecular markers associated with spermatogenesis may predict the pres- 
ence of mature spermatozoa in men with NOA $[24,25]$.

High levels of proliferative activity during spermatogenesis make germ cells more susceptible to DNA-damaging factors than other cells. More severe DNA damage caused by various endogenous and exogenous agents can result in germ cell loss and subsequent adverse outcomes, such as impaired spermatogenesis and male infertility [26]. In response to DNA damage, the phosphorylation of MDC1 causes RNF8 to be recruited to the locations of DNA double-strand breaks (DSBs), where RNF8 couples with Ubc13 catalyzes the construction of lysine 63-linked polyubiquitin chains on histones. The ubiquitination of histones at DSBs provides a fundamental chromatin structure for the subsequent function of proteins vital for DNA damage repair, such as 53BP1 and BRCA1 $[27,28]$.

Some molecular markers have previously been proposed; for example, ESX1 was identified in $95.4 \%$ of studied cases and showed a specificity of $74 \%$ and sensitivity of $80 \%$ for predicting residual spermatogenesis [29]. In a study by Ando et al. [22], the level of VASA transcription was evaluated using RT-qPCR in 52 samples from men with NOA, and it was demonstrated to be a predictive factor for sperm recovery, with $86.2 \%$ specificity and $87.0 \%$ sensitivity. In another study, HSFY was proposed as a potential diagnostic marker for sperm recovery in NOA patients, with a specificity of $92.6 \%$ and a sensitivity of $66.7 \%$ [23]. Despite the identification of these biomarkers, the quest to identify more accurate diagnostic markers is still continuing. Recently, histone demethylase JMJD1A, with a cutoff level of 0.74 and a specificity of $89.29 \%$ and a sensitivity of $90.91 \%$, demonstrated a more reliable predictive value for sperm recovery than the other previously suggested markers [30].

In the present study, the expression of RNF8 was evaluated in 40 NOA patients to assess the ability of the level of RNF8 expression to serve as a biomarker for the presence of mature spermatids. The expression of RNF8 at the transcript level was significantly higher in the NOA- group than in the NOA+ group. Moreover, the AUC of RNF8 expression for the entire study population was 0.84 , showing the discriminatory power of RNF8 expression for differentiating between the NOA- group and the NOA+ group. In the ROC curve analysis, RNF8 expression showed a sensitivity of $81 \%$ and a specificity of $84 \%$, with a cutoff level of 1.76 .

This study is the first to report the upregulation of RNF8 expression using RT-qPCR, However, a microarray study by Spiess et al. [31] showed significantly higher expression of RNF8 in individuals with Sertoli cell-only syndrome histology than in men with normal spermatogenesis. Moreover, a haplotype analysis of rs104669 and rs195432 of RNF8, which were in strong linkage disequilibrium, revealed that the $\mathrm{AC}$ haplotype was correlated with a reduced risk of NOA and smaller testis volume in a Chinese population [32]. Considering the elevated level of RNF8 expression in the patients with im- paired spermatogenesis in this study, this alteration likely reflects changes in somatic cells, which may imply that RNF8 has potential for interventional therapies [33].

Although our study suggests that RNF8 has potential as a novel biomarker for sperm recovery, some limitations are worth mentioning. First, sperm recovery based on mTESE is not sufficiently precise, because spermatogenesis is well known to exhibit heterogeneity in the testes of men with NOA, such that loci of spermatogenesis can coexist within Sertoli-only seminiferous tubules. Second, seminal plasma from azoospermic patients may be a better target for biomarker investigation, as it reflects more complex patterns of wholetestis physiology than mTESE samples, which only enable the analysis of a limited region of tissue. With these considerations in mind, it is recommended to utilize samples with high homogeneity and to promote the investigation of gene expression patterns as biomarkers in seminal plasma by focusing on finding biomarkers for sperm retrieval in NOA patients before performing mTESE.

This study presents a significant association between the expression of RNF8 and the presence of sperm in NOA patients, and therefore suggests that quantified RNF8 expression in testicular biopsy samples may be a valuable biomarker for predicting the presence of spermatozoa in biopsy samples.

\section{Conflict of interest}

No potential conflict of interest relevant to this article was reported.

\section{Author contributions}

Conceptualization: NG, MN, EB. Data curation: MN, EB, SMAZ. Formal analysis: SMAZ, MN, MT. Methodology: NG, NN, MS, MN, MD. Project administration: MN, NN, MS, MD. Writing - original draft: MN, MT. Writing - review \& editing: NG, EB, MN.

\section{References}

1. Gnoth C, Godehardt E, Frank-Herrmann P, Friol K, Tigges J, Freundl G. Definition and prevalence of subfertility and infertility. Hum Reprod 2005;20:1144-7.

2. Hamada A, Esteves SC, Nizza M, Agarwal A. Unexplained male infertility: diagnosis and management. Int Braz J Urol 2012;38:57694.

3. Ferlin A, Arredi B, Foresta C. Genetic causes of male infertility. Reprod Toxicol 2006;22:133-41.

4. Wosnitzer M, Goldstein M, Hardy MP. Review of azoospermia. Spermatogenesis 2014;4:e28218.

5. Silber SJ, Van Steirteghem AC, Devroey P. Sertoli cell only revisit- 
ed. Hum Reprod 1995;10:1031-2.

6. Hauser R, Botchan A, Amit A, Ben Yosef D, Gamzu R, Paz G, et al. Multiple testicular sampling in non-obstructive azoospermia: is it necessary? Hum Reprod 1998;13:3081-5.

7. Devroey P, Liu J, Nagy Z, Goossens A, Tournaye H, Camus M, et al. Pregnancies after testicular sperm extraction and intracytoplasmic sperm injection in non-obstructive azoospermia. Hum Reprod 1995;10:1457-60.

8. Plas E, Riedl CR, Engelhardt PF, Muhlbauer H, Pfluger H. Unilateral or bilateral testicular biopsy in the era of intracytoplasmic sperm injection. J Urol 1999;162:2010-3.

9. Vernaeve V, Verheyen G, Goossens A, van Steirteghem A, Devroey $P$, Tournaye $H$. How successful is repeat testicular sperm extraction in patients with azoospermia? Hum Reprod 2006;21:1551-4.

10. Tsujimura A, Matsumiya K, Miyagawa Y, Takao T, Fujita K, Koga M, et al. Prediction of successful outcome of microdissection testicular sperm extraction in men with idiopathic nonobstructive azoospermia. J Urol 2004;172(5 Pt 1):1944-7.

11. Talas H, Yaman O, Aydos K. Outcome of repeated micro-surgical testicular sperm extraction in patients with non-obstructive azoospermia. Asian J Androl 2007;9:668-73.

12. Feng $L$, Chen J. The E3 ligase RNF8 regulates KU80 removal and NHEJ repair. Nat Struct Mol Biol 2012;19:201-6.

13. Mallette FA, Mattiroli F, Cui G, Young LC, Hendzel MJ, Mer G, et al. RNF8- and RNF168-dependent degradation of KDM4A/JMJD2A triggers 53BP1 recruitment to DNA damage sites. EMBO J 2012; 31:1865-78.

14. Baarends WM, Hoogerbrugge JW, Roest HP, Ooms M, Vreeburg J, Hoeijmakers $\mathrm{JH}$, et al. Histone ubiquitination and chromatin remodeling in mouse spermatogenesis. Dev Biol 1999;207:32233.

15. Meistrich ML, Mohapatra B, Shirley CR, Zhao M. Roles of transition nuclear proteins in spermiogenesis. Chromosoma 2003;111: 483-8.

16. Ma T, Keller JA, Yu X. RNF8-dependent histone ubiquitination during DNA damage response and spermatogenesis. Acta Biochim Biophys Sin (Shanghai) 2011;43:339-45.

17. Santos MA, Huen MS, Jankovic M, Chen HT, Lopez-Contreras AJ, Klein IA, et al. Class switching and meiotic defects in mice lacking the E3 ubiquitin ligase RNF8. J Exp Med 2010;207:973-81.

18. Sin HS, Barski A, Zhang F, Kartashov AV, Nussenzweig A, Chen J, et al. RNF8 regulates active epigenetic modifications and escape gene activation from inactive sex chromosomes in post-meiotic spermatids. Genes Dev 2012;26:2737-48.

19. Mueller JL, Mahadevaiah SK, Park PJ, Warburton PE, Page DC, Turner JM. The mouse $X$ chromosome is enriched for multicopy testis genes showing postmeiotic expression. Nat Genet 2008;
40:794-9.

20. Toure A, Szot M, Mahadevaiah SK, Rattigan A, Ojarikre OA, Burgoyne PS. A new deletion of the mouse $Y$ chromosome long arm associated with the loss of Ssty expression, abnormal sperm development and sterility. Genetics 2004;166:901-12.

21. Ben-Yosef D, Yogev L, Hauser R, Yavetz H, Azem F, Yovel I, et al. Testicular sperm retrieval and cryopreservation prior to initiating ovarian stimulation as the first line approach in patients with non-obstructive azoospermia. Hum Reprod 1999;14:1794-801.

22. Ando M, Yamaguchi K, Chiba K, Miyake H, Fujisawa M. Expression of VASA mRNA in testis as a significant predictor of sperm recovery by microdissection testicular sperm extraction in patient with nonobstructive azoospermia. J Androl 2012;33:711-6.

23. Stahl PJ, Mielnik A, Schlegel PN, Paduch DA. Heat shock factor $Y$ chromosome (HSFY) mRNA level predicts the presence of retrievable testicular sperm in men with nonobstructive azoospermia. Fertil Steril 2011;96:303-8.

24. Bonaparte E, Moretti M, Colpi GM, Nerva F, Contalbi G, Vaccalluzzo L, et al. ESX1 gene expression as a robust marker of residual spermatogenesis in azoospermic men. Hum Reprod 2010;25: 1398-403.

25. Kleiman SE, Lehavi O, Hauser R, Botchan A, Paz G, Yavetz H, et al. CDY1 and BOULE transcripts assessed in the same biopsy as predictive markers for successful testicular sperm retrieval. Fertil Steril 2011;95:2297-302.

26. Gunes S, Al-Sadaan M, Agarwal A. Spermatogenesis, DNA damage and DNA repair mechanisms in male infertility. Reprod Biomed Online 2015;31:309-19.

27. Kolas NK, Chapman JR, Nakada S, Ylanko J, Chahwan R, Sweeney $\mathrm{FD}$, et al. Orchestration of the DNA-damage response by the RNF8 ubiquitin ligase. Science 2007;318:1637-40.

28. Mailand N, Bekker-Jensen S, Faustrup H, Melander F, Bartek J, Lukas C, et al. RNF8 ubiquitylates histones at DNA double-strand breaks and promotes assembly of repair proteins. Cell 2007;131: 887-900.

29. Pansa A, Sirchia SM, Melis S, Giacchetta D, Castiglioni M, Colapietro $P$, et al. ESX1 mRNA expression in seminal fluid is an indicator of residual spermatogenesis in non-obstructive azoospermic men. Hum Reprod 2014;29:2620-7.

30. Eelaminejad Z, Favaedi R, Modarresi T, Sabbaghian M, Sadighi Gilani MA, Shahhoseini M. Association between JMJD1A expression and sperm retrieval in non-obstructive azoospermic patients. Cell J 2018;19:660-5.

31. Spiess AN, Feig C, Schulze W, Chalmel F, Cappallo-Obermann H, Primig $M$, et al. Cross-platform gene expression signature of human spermatogenic failure reveals inflammatory-like response. Hum Reprod 2007;22:2936-46. 
32. Zhang Y, Song B, Du WD, He XJ, Ruan J, Zhou FS, et al. Genetic association study of RNF8 and BRDT variants with non-obstructive azoospermia in the Chinese Han population. Syst Biol Reprod Med 2015;61:26-31.
33. He Z, Chan WY, Dym M. Microarray technology offers a novel tool for the diagnosis and identification of therapeutic targets for male infertility. Reproduction 2006;132:11-9. 